\title{
A note on black hole entropy, area spectrum, and evaporation
}

\author{
C.A.S.Silva ${ }^{\text {* }}$ and R.R. Landin円 \\ Departamento de Física, Universidade Federal do Ceará - Caixa Postal 6030, CEP 60455-760, Fortaleza, Ceará, Brazil
}

\begin{abstract}
We argue that a process where a fuzzy space splits in two others can be used to explain the origin of the black hole entropy, and why a "generalized second law of thermodynamics" appears to hold in the presence of black holes. We reach the Bekenstein-Hawking formula from the count of the microstates of a black hole modeled by a fuzzy space. In this approach, a discrete area spectrum for the black hole, which becomes increasingly spaced as the black hole approaches the Planck scale, is obtained. We show that, as a consequence of this, the black hole radiation becomes less and less entropic as the black hole evaporates, in a way that some information about its initial state could be recovered.
\end{abstract}

It is not an exaggeration to say that one of the most exciting predictions of general relativity is that there may exist black holes. This is mainly due to the believe that black holes may play a major role in our attempts to shed some light on the nature of a quantum theory of gravity such as the role played by atoms in the early development of quantum mechanics.

Recent results have shown that black hole have thermodynamics properties like entropy and temperature, and as a consequence of the instability of the vacuum in strong gravitational fields, black holes are sources of quantum radiation [1,3].

Some time after, string theory and loop quantum gravity, argued that the origin of the black hole entropy must be related with the quantum structure of the spacetime [4, 5]. However the computation of black hole entropy in the semiclassical and furthermore in quantum regime has been a very difficult, and still unsolved problem. We know that in statistical physics, entropy counts the number of accessible microstates that a system can occupy, where all states are presumed to occur with equal probability. On the other hand, we also know that black holes can be completely characterized by only three externally observable classical parameters: mass, electric charge, and angular momentum. All other information about the matter which formed a black hole "disappears" behind the black-hole event horizon, and therefore the nature of these microstates is obscure. Then, what is the origin of the black hole entropy? Furthermore, in order to justify the name "entropy", one must to explain also why $S=S_{b h}+S_{\text {out }}$ is a non-decreasing function of time, in other words, why black holes obey a "generalized second law of thermodynamics".

We still have that, since black holes evaporate, one could expect, from the Hawking radiation, any information about the state which collapsed into the black hole. However, Hawking showed that this radiation is thermal, and therefore does not carry any information about the black hole initial state. That is to say, no information can escape from inside of the black hole horizon. In this

\footnotetext{
*Electronic address: calex@fisica.ufc.br
}

† Electronic address: renan@fisica.ufc.br situation, the matter that formed the black hole, which initially was in a pure state has evolved into a mixed state. This bring us a contradiction with quantum mechanics. There, a pure state can only evolve into another pure state because of the unitarity of the evolution operator [1, 2, 6, 7].

One way to solve the Hawking paradox is that where information can be stored in a topological disconnected region which arises inside of the black hole [10]. Gravitational collapse would lead to a region of Planckian densities and curvature where quantum gravitational effects can lead to a topology change process where a new topologically disconnected region appears. However, This process had been claim to break unitarity and cluster deposition(locality) [10, 11.

On the other hand, recently it was suggested by one of us that it is possible to realize a topology change process to black holes without break unitarity or cluster decomposition 9. The basic idea of this proposal is to see the black hole horizon as a fuzzy sphere $S_{F}^{2}$ taking into account some quantum symmetry properties related with a Hopf algebra structure. There, we can define a linear operation (the coproduct of the Hopf algebra) on $S_{F}^{2}$ and compose two fuzzy spheres preserving algebraic properties intact. This operation, which we shall represent by $\Delta$, produces a topology change process where a fuzzy sphere splits in two others [12.

Let M describes a wave function on $S_{F}^{2}$, the coproduct $\Delta: S_{F}^{2}(J) \rightarrow S_{F}^{2}(K) \otimes S_{F}^{2}(L)$ acts on $\mathrm{M}$ as

$$
\begin{aligned}
\Delta(M)_{(K, L)} & =\sum_{\mu_{1}, \mu_{2}, m_{1}, m_{2}} C_{K, L, J ; \mu_{1}, \mu_{2}} C_{K, L, J ; m_{1}, m_{2}} \\
& \times M_{\mu_{1}+\mu_{2}, m_{1}+m_{2}} e^{\mu_{1} m_{1}}(K) \otimes e^{\mu_{2} m_{2}}(L)(1)
\end{aligned}
$$

where $C$ 's are the Clebsh-Gordan coefficients and $e^{\mu_{i} m_{j}}$ 's are basis for Mat. From (1) $\mathrm{M} \in S_{F}^{2}(J)$ splits into a superposition of wavefunctions on $S_{F}^{2}(K) \otimes S_{F}^{2}(L)$, and the information in $M$ is divided between the two fuzzy spheres with spins $K$ and $L$ respectively.

The process (1) preserves the Hermitian conjugation, the matrix product, the trace, and the inner product.The two last properties assure that (1) is a unitary process 12 .

Following [9], if we use the fuzzy sphere Hilbert space as the ones of the black hole, we shall have the following 
consequences: (i) The maximum of information about the black hole that an outside observer can obtain would be encoded in a wave function $M$ defined on the fuzzy sphere Hilbert space. (ii) We shall find out, through the Hopf coproduct $\Delta$ in (1), a topology change process for the black hole. In this process the information about the black hole initial state, described by the wave function $M$, is divided into two regions. One of them is a fuzzy sphere with spin $K$, which we shall consider as the original world and name it "the main world". The other one is a fuzzy sphere with spin $L$ which we shall name "the baby world". (iii) Since an observer in the main world can not access the degrees of freedom in the baby world, from his standpoint, the black hole will appear to evolve from a pure to a mixed state, described by a density matrix $\rho$. Therefore, we can define an entropy measured by the observer in the main world.

The entropy measured by the observer in the main world would be given by $S=-k_{B} \ln (\rho \operatorname{tr} \rho)=$ $k_{B} \ln (\operatorname{dim} H)$, where $H$ is Hilbert space associated to the black hole. If we associate a Hilbert space $H_{i}$ with each cell on the fuzzy sphere, we shall have that, for a fuzzy sphere with $\mathrm{n}$ cells, the hole Hilbert space will be given by $H=\bigotimes_{i=1}^{n} H_{i}$. Therefore, we shall have $S=k_{B} \ln \left(\operatorname{dim}_{i}\right)^{n}=n k_{B} \ln \left(\operatorname{dimH}_{i}\right)$. On the other hand, the fuzzy sphere area can be written as $A=\alpha n l_{P}^{2}$, where $\alpha$ is related with the quantization scale, and $l_{P}^{2}$ is the Planck area.

In this way, we have that the black hole entropy can be written as

$$
S=\varepsilon k_{B} \frac{A}{l_{P}^{2}}
$$

which corresponds to the Bekenstein-Hawking formula, unless for the undetermined factor $\varepsilon=\ln \left(\operatorname{dim} H_{i}\right) / \alpha$.

The quantum topology change model, in this way, shed some light on the problem of the origin of the entropy associated to a black hole by an outside observer: it is generated because of the non-unitary evolutions of the geometry of the main world. As we shall show, the nonunitary evolution of the black hole geometry can also be the origin of the GSL.

Now let us address the black hole evaporation process, as it is seen by an observer in our universe, through the topology change (1). In the intend to do this, at first, let us address the black hole area spectrum. From the equation 2, this spectrum is given by

$$
A_{j}=\epsilon^{-1} l_{p}^{2} \ln (2 j+1) .
$$

We can choose the splitting process (1) in a way that $j \rightarrow j-\frac{1}{2}$, in the main world, and from $(3)$ this it will result in a decrease of the black hole area, until when $j=0$ it has completely evaporated. The logarithmic dependence of the black hole area spectrum on $j$ in the expression (3) tell us that, the decrease in the horizon area occurs in a continuous way to large values of $j$, and in a discrete way to small values of $j$, when the black hole approaches the Planck scale, as have been shown in the figure 1 .

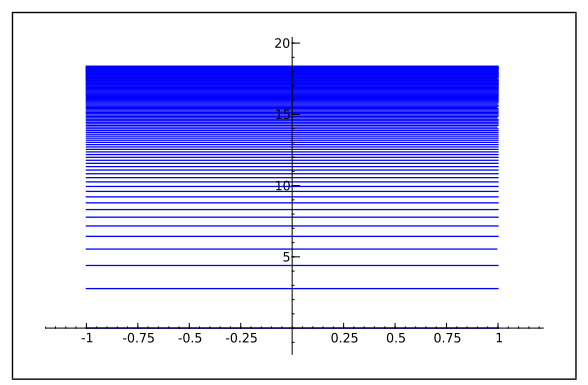

FIG. 1: Area spectrum, in units of Planck area for $\epsilon=1 / 4$, to a quantum black hole in topology change approach. From the logarithmic dependence of the black hole area spectrum on $j$ in (3), we have that the levels become continuous for larger area values.

We have that the Hawking radiation is known semiclassically to be continuous. However, the Hawking quanta of energy are not able to hover at a fixed distance from the horizon since the geometry of the horizon has to fluctuate, once quantum gravitational effects are included. Thus, one suspects a modification of the black hole radiation, when quantum geometrical effects are taken into account. Then any modification on the description of the black hole emission process must occur at the final stages of black hole evaporation, where its area spectrum becomes discrete.

To address it, let us analyze the process which consists in to go one step down in the black hole area spectrum $(j \rightarrow j-1 / 2)$. From equation (1), we have that, tracing over the degrees of freedom in the baby universe, the splitting process (1), for a matrix $M=|j, m\rangle\left\langle j, m^{\prime}\right|$ with $l=1 / 2$, and $k=j-1 / 2$ is given by:

$$
\begin{aligned}
\Delta\left(|j, m\rangle\left\langle j, m^{\prime}\right|\right) \quad= \\
\quad \frac{\sqrt{\left(k+m_{k}+1\right)\left(k+m_{k}^{\prime}+1\right)}}{2 k+1}|k, m-1 / 2\rangle\left\langle k, m^{\prime}-1 / 2\right| \\
+\frac{\sqrt{\left(k-m_{k}+1\right)\left(k-m_{k}^{\prime}+1\right)}}{2 k+1}|k, m+1 / 2\rangle\left\langle k, m^{\prime}+1 / 2\right| .
\end{aligned}
$$

In this way, the probability to go down one step in the black hole area spectrum is given by

$$
p_{j \rightarrow k=j-1 / 2}=\text { phase } \times\left(\frac{2 k+2}{2 k+1}\right)=\text { phase } \times e^{\frac{-\epsilon \delta A}{l_{p}^{2}}},
$$

where a normalization factor $(2 k+1)^{-1}$ has been included.

The probability for a black hole goes n steps down into its area spectrum can be obtained from equation (4). In reference 9] it been shown that the splitting process (1) obeys cluster decomposition. In this way, we have that 
the different steps $j \rightarrow j-1 / 2$ in the black hole evaporation are independent events. Then the probability of $n$ steps occur in the black evaporation process is given by the product of the probabilities of each one of this steps occurring by itself. Then we have,

$$
p_{k n}=\text { phase } \times\left(\frac{2 k+2}{2 k-n+1}\right)=\text { phase } \times e^{\frac{-\epsilon \delta A_{k n}}{l_{p}^{2}}}
$$

The probability above depends on the undetermined parameter $\epsilon$, which appears in the expression to the black hole entropy (2). We have that, from the equation (5), the density matrix describing the black hole is given by $\hat{\rho} \sim \sum_{j} e^{-\epsilon A_{j}}|j\rangle\langle j|=e^{-\epsilon \hat{A}}$. In this way, the black hole density matrix must satisfy the equation

$$
i \frac{\partial \hat{\rho}}{\partial \Theta}=-\frac{\hat{A}}{8 \pi} \hat{\rho}
$$

As addressed by 14 and [15, the parameter $\Theta=8 \pi i \epsilon$ plays the role of a sort of "dimensionless internal time" associated with the horizon. The equation (6) must be used when working in the Euclidean continuation of the black hole [13. Regularity of the Euclidean manifold at the horizon imposes a fixed Euclidean angle given by $\Theta=$ $2 \pi i$ [13], 14], and [15. In this way, the undetermined parameter in (2) and (5) is fixed as $\epsilon=1 / 4$.

The results above revel the evolution of the black hole geometry induced by the topology change process (1) in the evaporation process, and bring essential consequences for the way how entropy is emitted in the black hole evaporation process.

The entropy of a system measures one's lack of information about its actual internal configuration [16 19]. Suppose that all that is known about the internal configuration of the system is that it may be found in any of a number of states, with probability $p_{n}$ for the nth state. Then the entropy associated with the system is given by Shannon's well-known relation $S=-\sum p_{n} \ln p_{n}$.

The probability for a black hole to emit a specific quantum should be given by the expression (5), in which we shall include a gray-body factor $\Gamma$ (representing a scattering of the quantum off the spacetime curvature surround the black hole). Thus, the probability $p_{n}$ to $\mathrm{n}$ steps in the mass (area) ladder is proportional to $\Gamma(n) e^{-\frac{\delta A_{k n}}{4 l_{p}^{2}}}$.

The discrete mass (area) spectrum (3) implies a discrete line emission from a quantum black hole. For a Schwarzschild black hole, the radiation emitted by the black hole will be at frequencies given by $\omega=$ $\frac{1}{2 \sqrt{\pi}}(\sqrt{\ln (2 j+1)}-\sqrt{\ln (2 j+1-n)})$.

To gain some insight into the physical problem, we shall consider a simple toy model suggested by Hod [20. It is well known that, for massless field, $\Gamma(M \omega)$ approaches 0 in the low-frequency limit, and has a highfrequency limit of 1 21 23. That is, $\Gamma(\bar{\omega})=0$ for $\bar{\omega}<\bar{\omega}_{c}$, and $\Gamma(\bar{\omega})=1$ otherwise, where $\bar{\omega}=M \omega$.
The ratio $R=\left|\dot{S}_{\text {rad }} / \dot{S}_{B H}\right|$ of entropy emission rate from the quantum black hole to the rate of black hole entropy decrease is given by:

$$
R=\frac{-\sum_{i=1}^{N_{s}} \sum_{n=1}^{2 k+1} C \Gamma(n) e^{-\frac{\delta A_{k n}}{4}} \ln \left[C \Gamma(n) e^{-\frac{\delta A_{k n}}{4}}\right]}{\sum_{i=1}^{N_{s}} \sum_{n=1}^{2 k+1} C \Gamma(n) e^{-\frac{\delta A_{k n}}{4}}\left(\frac{\delta A_{k n}}{4}\right)}
$$

where $\mathrm{C}$ is a normalization factor, defined by the normalization condition:

$$
\sum_{i=1}^{N_{s}} \sum_{n=1}^{2 k+1} C \Gamma(n) e^{-\frac{\delta A_{k n}}{4}}=1
$$

$N_{s}$ is the effective number of particle species emitted ( $N_{s}$ takes into account the various modes emitted). We shall consider $N_{s} \propto 2 k+2$, with a proportionality constant less than or equal to one, since the modes emitted by the black hole in our treatment must be upper limited by the number of degrees of freedom on the fuzzy sphere.

We have plotted $\mathrm{R}$ down, taking $\bar{\omega}_{c} \simeq 0.2$ (the location of the peak in the total power spectrum [21 23]).

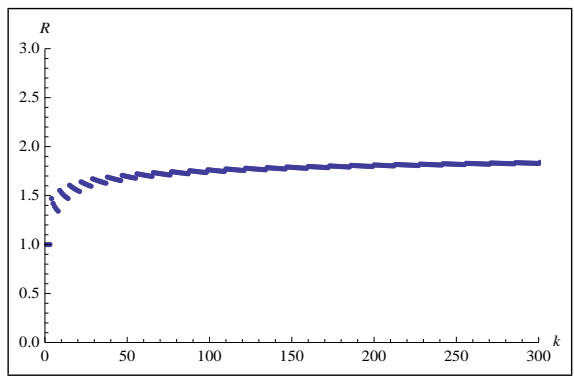

From the graphic above, we have that the emission process respect a "second law of thermodynamics", since $\mathrm{R}$ is ever larger than (or equal) to unity. In this way, the non-unitary evolution of the black hole geometry in the main world, due to the topology change process, can be the origin of the GSL.

Besides it is important to notice the entropy emitted from the black hole decreases as the area spacing increases. In this way, the entropy of the radiation emitted by the black hole becomes increasingly smaller with each step of the evaporation process, mainly when the black hole reaches the Planck scale (notice that $\mathrm{R}$ reaches the unity in the last steps).

In this work we have argued that the quantum topology change model to black hole evaporation, proposed by us in reference 9, shed some light on the problem of the origin of black hole entropy: it is generated because of the non-unitary evolutions of the geometry of the main world. Besides, the topology change approach give us a relation of states to points that agrees with our standard concept of entropy as the logarithm of the number of microstates, from which we have derived the BekensteinHawking formula, $S=A / 4$. 
From the topology change model we have obtained a black hole area spectrum, which is continuous in the classical/semiclassical limit, and becomes discrete as the black hole approaches the Planck scale. As a consequence of this, we have that information can leak out from black hole, since its radiation becomes less and less entropic as the black hole evaporates. In this way, some information about the black hole initial state could be accessible to an observer in our universe. Since it would occurs more strongly in the quantum gravity limit, it does not require radical modifications in the laws of physics above the Planck scale. The task of found an appropriate quantum mechanism for information leak remains.

We have also shown that the description of the black hole evaporation through a quantum topology change process proposed by [9] could be the origin of the GSL.

The authors thanks to Coordenação de Aperfeioamento de Pessoal de Ensino Superior-CAPES(Brazil), and Conselho Nacional de Desenvolvimento Cientfico e Tecnolgico - CNPQ(Brazil) for the financial support, and C.A.S. Almeida for the careful reading of the manuscript
[1] S.W. Hawking, Particle Creation by Black Holes, Commun.Math.Phys. 43 (1975) 199-220.

[2] S.W. Hawking, Breakdown of Predictability in Gravitational Collapse, Phys.Rev.D 14 (1976) 2460-2473.

[3] J.D. Bekenstein, Black holes and the second law, Lett. Nuovo Cim. 4 (1972) 737-740.

[4] Andrew Strominger and Cumrun Vafa, Microscopic origin of the Bekenstein-Hawking entropy , Phys. Lett. B 379 (1996) 99-10.

[5] A. Ashtekar, J. Baez, A. Corichi, and K. Krasnov, Quantum Geometry and Black Hole Entropy, Phys. Rev. Lett. 80, (1998) 904-907.

[6] J. Preskill, Do black holes destroy information?, hepth/9209058.

[7] D.N. Page, Black hole information, hepth/9305040.

[8] J. Madore, The Fuzzy sphere, Class.Quant.Grav. 9 (1992) 69-88.

[9] C.A.S. Silva, Fuzzy spaces topology change as a possible solution to the black hole information loss paradox, Phys.Lett.B 677 (2009), 318-321.

[10] S.D.H. Hsu, Spacetime topology change and black hole information, Phys.Lett.B 664 (2007) 67-71.

[11] L. Susskind, Comment on a proposal by Strominger, hepth/9405103

[12] A.P. Balachandran, S. Kürkçüoğlu, Topology change for fuzzy physics: Fuzzy spaces as Hopf algebras, Int.J.Mod.Phys.A 19 (2004) 3395-3408.

[13] M. Banados, C. Teitelboim, J. Zanelli, Black hole entropy and the dimensional continuation of the Gauss-Bonnet theorem, Phys.Rev.Lett.72 (1994) 957-960.

[14] Steven Carlip, Claudio Teitelboim, The off-shell black hole, Class.Quant.Grav.12 (1995) 1699-1704.

[15] S. Massar, R. Parentani, How the change in horizon area drives black hole evaporation, Nucl.Phys.B 575 (2000) 333-356.

[16] C.E. Shannon and W. Weaver, The mathematical theory of communications (University of Illinois Press, Urbana, 1949).

[17] E.T. Jaynes, Information Theory and Statistical Mechanics, Phys.Rev.106 (1957) 620-630.

[18] E.T. Jaynes, Information Theory and Statistical Mechanics II, Phys.Rev.108 (1957) 171-190.

[19] J. D. Bekenstein, Black Holes and Entropy, Phys. Rev. D 7 (1973) 2333-2346.

[20] Shahar Hod, Black hole radiation, the fundamental area unit, and the spectrum of particle species, Phys.Rev.D 61 (2000) 124016.

[21] D.N. Page, Particle Emission Rates from a Black Hole: Massless Particles from an Uncharged, Nonrotating Hole, Phys.Rev.D13 (1976) 198-206.

[22] D.N. Page, Particle Emission Rates from a Black Hole. 2. Massless Particles from a Rotating Hole, Phys.Rev.D14 (1976) 3260-3273.

[23] D.N. Page, Particle Emission Rates from a Black Hole. 3. Charged Leptons from a Nonrotating Hole, Phys.Rev.D16 (1977) 2402-2411. 